\title{
A Study on Data Modeling Approach Utilizing 3-tier Architecture of 'BEE (Being-Experience-Event)'
}

\author{
Chul Hyun Hwang ${ }^{1}$, and Iek Jae Son ${ }^{1}$ and Hoe Kyung Jung ${ }^{2 *}$ \\ ${ }^{1}$ Director, eINSS\&C, 1-1501, Ace High City, 55-20, Mullae-Dong 3ga, \\ Seoul, South Korea \\ ${ }^{1}$ Chief of Naval Central Computing Center, Naval Central Computing Center, \\ South Korea \\ ${ }^{2}$ Dept. Of Computer Engineering, PciChai Univ., 155-40 Baejae-ro, Seo-gu, Daejeon, \\ South Korea \\ ${ }^{1}$ chhwang@einssnc.com, ${ }^{1}$ ahson2@naver.com, ${ }^{2}$ hkjung@pcu.ac.kr
}

\begin{abstract}
This study proposes BEE (Being Experience Event) approach as a feasible solution to the data sharing problem in e-Government environment. The proposed approach provides criteria for categorizing entities into three different layers and procedures that are required to achieve the goal of data sharing during data modeling process. The data model built based on the BEE approach helps achieve the goals of data sharing in an environment where multiple concept domains are present.
\end{abstract}

Keywords: Data Modeling, Data Sharing, Data Architecture, e-Government, Gov 3.0, Open Data

\section{Introduction}

The e-Government puts a great deal of emphasis on the values of inter-operability and collaboration within the government. The essential prerequisites to build an integrated and cooperative information system are information sharing and data $[2,3,8]$. In recent years, the government has evolved into Government 3.0 that opens public data to private sector to create new value added $[6,9]$.

The objective of the study is to propose a methodology for designing data structure that allows the data created for an internal task of a government organization to be used in the private sector as well as in other government organizations.

This paper is organized as follows: In Chapter 2, literature reviews on the necessity of the data sharing and solutions to the problems related to it are presented. In Chapter 3, the procedures of the proposed BEE approach and the criteria of the three layers are presented. In Chapter 4, the effectiveness of the proposed approach is validated by experiments. Conclusions and future works are presented in Chapter 5.

\section{Literature Review}

Pin et al. claims that data modeling on an enterprise schema level is necessary to overcome the limitations of user schema [7]. Their claim has been widely accepted since the data

\footnotetext{
${ }^{*}$ Corresponding Author
} 
modeling is fundamentally subjective and has the range and level limitations of a specific perspective.

However, since the data sharing problem of the e-Government arises in the relationship between different enterprise schemas, the ideal solution is to utilize ontology [4]. The ontology is based on the common sense that identifies a specific entity that exists in the real world [8]. In other words, the universal and objective recognition of the real world entity is the solution to the data sharing problem.

\section{BEE Approach}

\subsection{Limitations of existing studies}

As mentioned previously, in order for government 3.0 to achieve the goals of data sharing, following issues need to be addressed.

First, since the recent e-Government shares data with various stakeholders, and intends to re-use the data, the highest unity concept such as the enterprise view can not be realized. Another reason is that the e-Government shares the data that are present in various enterprise areas horizontally [5].

Second, the data intended for sharing are determined based on the concept approved by all stakeholders. However, the data used in the e-Government are shared not only within the government branches and public sector but also outside the boundaries of the nation. Therefore, an agreement can not be reached as it is impossible to define the range of stakeholders when an information system or a data model is being built. Open Data (or Open Government Data) is a typical example that exhibits this phenomenon.

Third, if the ontology is defined based on the sharing and agreement, distorted definition of the ontology becomes problematic when it is interpreted by a third party that has not participate in the process of agreement. The definition of ontology is not about the sharing and agreement but about being [10].

Fourth, existing studies propose methods to convert ontology into a relational model [1]. However, in order to secure sufficient open data and realize the e-Democracy, solving the data sharing problem itself through the relational model is the most effective method.

\subsection{Approach to solve data sharing problem}

In order to solve the data sharing problem, following conceptual approaches are necessary. The existence of matters and phenomena in real world is entirely different from explaining their existence. It must be made clear that the data is the latter because the explanation of the existence differs substantially depending on the viewpoint, situation or timing of the observer.

The e-Government is an environment where previously mentioned diversity exists in an extremely complex manner. A same social phenomenon can be viewed from an entirely different perspective, and it is possible to have multiple explanations for a specific fact. In this case, data exists only when the layer that proves its existence (being) and the layer that recognizes it (being experienced or explained) are separated. 'Being' is a criterion for sharing and 'being experienced' is a subject of sharing.

Merging of the two phases (single entity) is only allowed in limited cases where they are recognized and agreed upon by every member in a unified concept environment. This has an advantage that a simple explanation exists for a data model. However, it also isolates the data model from different external concept domains.

In summary, the information system is built based on the requirements of the user. During this process, it is problematic that the modeler often misinterprets interviews or references as the nature or the entirety of the real world. However, the value of the data shared by the e- 
Government lies where the data is utilized outside of the model and new knowledge is created.

\subsection{Introduction to BEE approach}

BEE approach classifies entities into three different categories namely Being, Experience and Event entities. First, the Being entity is a subject of de-concept and objectivity. The subjects are the objects in real world that are free from the categorical limit of a specific concept and considered to have the same meaning. Second, the Experience entity is a subject of concept and subjectivity. It means the subjective meaning or the information regarding the role of the entity that is determined by the experience of the observer. Third, the Even entity represents a specific incident or phenomenon.

\subsection{Procedures of BEE approach}

BEE approach consists of following three steps.

Step 1. Entities are drawn from the user requirements. In this step, the method of drawing is based on the traditional relational database modeling technique.

Step 2. The entities are categorized based on the previously mentioned BEE classification method. Additional entities that are not drawn from the user requirements are drawn based on the results of the classification. However, it is highly possible that the data categorized originally as the Being layer in step 1 is in fact the Experience layer because it is merely a subjective term that is agreed upon by all stakeholders within the concept domain.

Step 3. The relationship between the entities is established by the criteria of the categorization. Any entity in the Event layer must refer to one or more entities in the Experiment layer, and any entity in the Experiment layer to one or more entities in the Being layer.

A distinguishing feature of the BEE approach is that it finds the missing entities from the user requirements and visualizes business rules. An example of the data model based on the BEE approach is shown in Figure 1.
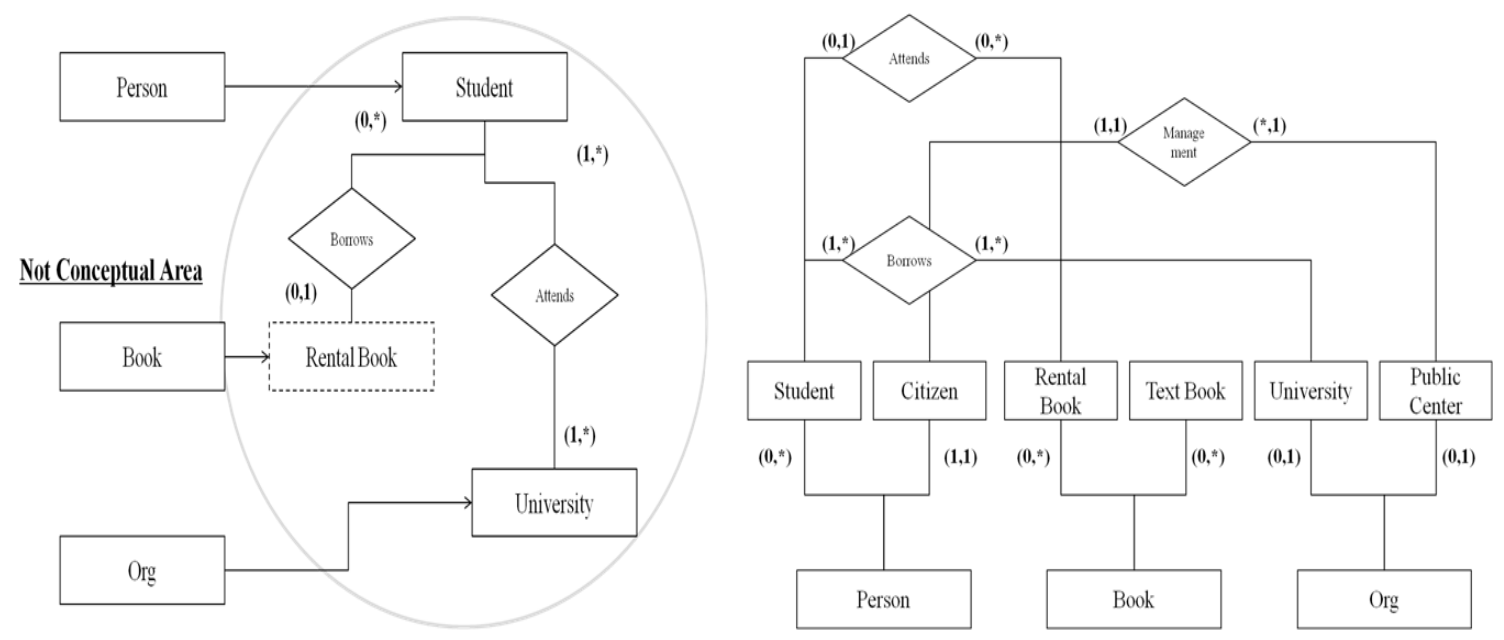

Figure 1. example of the data model based on the BEE approaches 


\section{Results and Validation}

\subsection{Selecting experimental data}

The experimental data is selected from the answer sheets collected for four years (20092012) in a DA contest offered to the general public, which is hosted by KoDB (Korea Data Base agency). Reasons for selecting them as the experiment target are as follows.

First, the contest is a data model that is intentionally applied to various domain areas. The contest provides data for various areas every year including the public and the private sectors.

Second, it is easy to compare as they provides outcome from a data model that is fully compliant with traditional approaches.

Third, as requirements are preliminarily fixed, the level of conceptual modeling is obvious.

Descriptions, areas of subjects and the number of requirements for 4 information systems used in the experiment are stated in the Table 1 below. Each experiment target is selected through independent process.

\section{Table 1. Conceptual Areas and Number of Requirements for Experiment Targets}

The number of requirements is based on the number of articles specified in the requirement specifications

\begin{tabular}{|c|l|c|}
\hline Case & \multicolumn{1}{|c|}{ Description } & $\begin{array}{c}\text { Number of } \\
\text { Requirements }\end{array}$ \\
\hline 1 & $\begin{array}{l}\text { Performs the research project support and project management for public } \\
\text { institutions (2009) }\end{array}$ & 68 \\
\hline 2 & $\begin{array}{l}\text { Performs project management for SI company } \\
\text { (2010, outsourcing, schedule, outcome, process control) }\end{array}$ & 53 \\
\hline 3 & Performs disposition of construction waste for local government (2011) & 43 \\
\hline 4 & $\begin{array}{l}\text { Performs order control for a furniture company specialized in custom } \\
\text { manufacture and sales of finished products (2012) }\end{array}$ & 70 \\
\hline
\end{tabular}

\subsection{Results}

The experiment is conducted based on the six scenarios constructed from four subjects. The main objective of the experiment is to verify whether the proposed BEE approach can increase the level of data sharing in various information system building environments.

According to the results, the BEE approach increases both the number of entities and the rate of sharing compared to the traditional modeling techniques as shown in Figure 2. It is remarkable that the BEE approach increases the rate of sharing for the same subject of the modeling even though the number of entities is increased. The reason for this behavior is that the added entities play an essential role in increasing the rate of the sharing. 


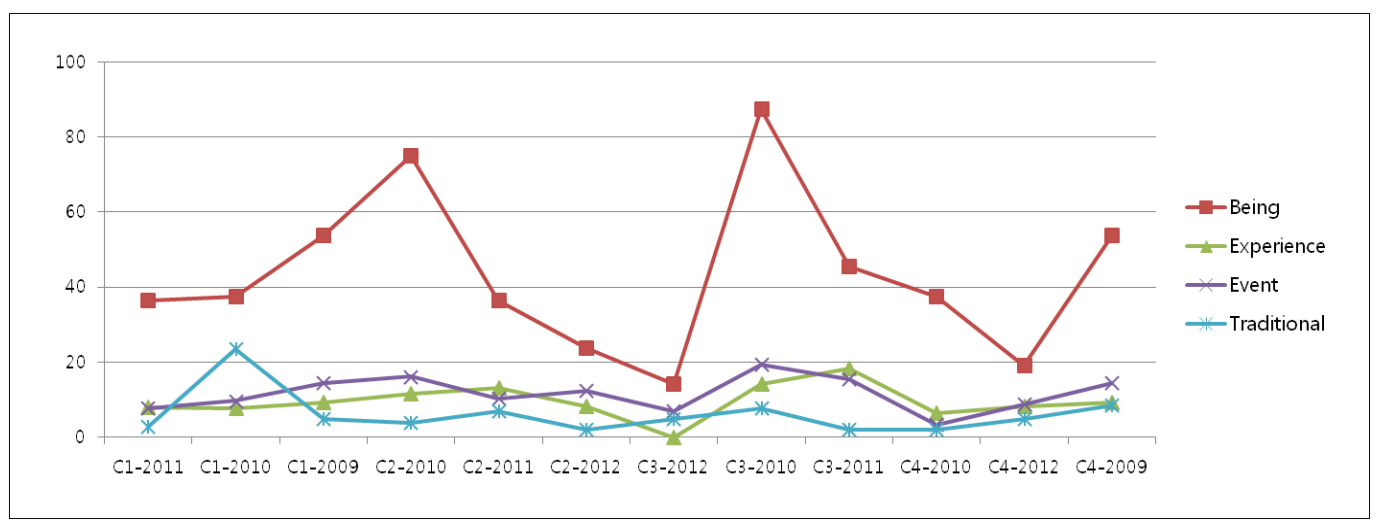

Figure 2. Conceptual Areas of Experiment Target and Number of Requirements

The experimental results that support this claim are shown in Figure 3. Compared to the entities drawn directly from the user requirements, the additional entities drawn from the BEE approach show significant increase in the rate of reuse.

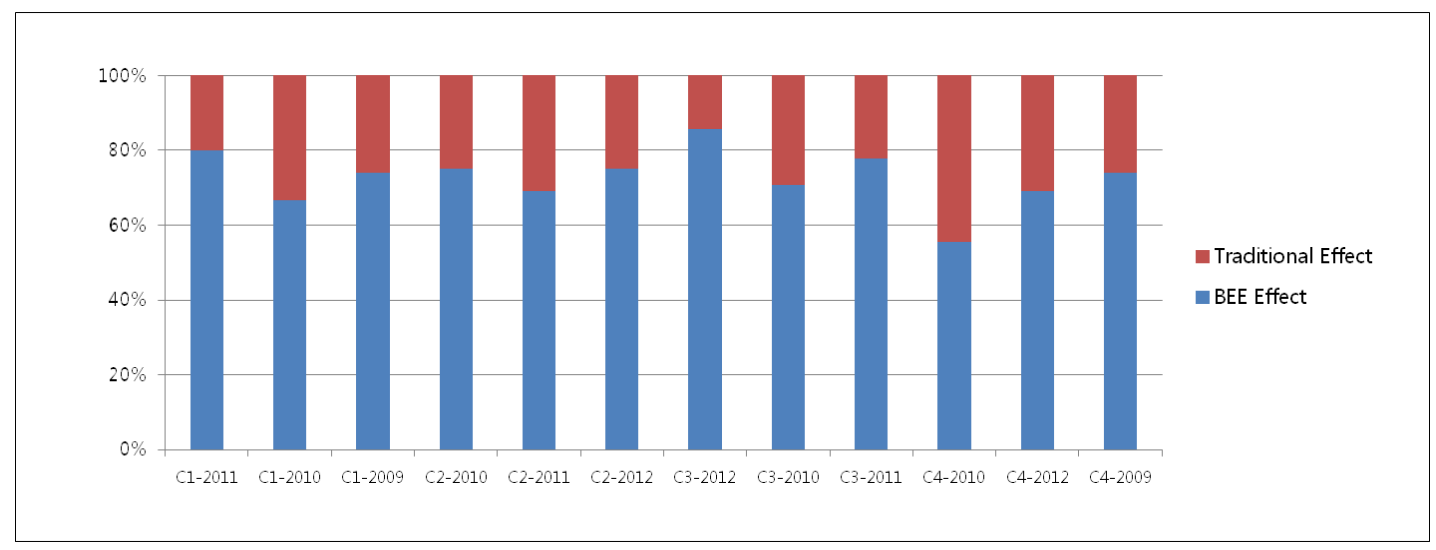

Figure 3.Comparing the approach effect

The experimental results show that finding the entities that are missed by the user requirements and managing the data based on the BEE approach are important processes in the data sharing.

\section{Conclusions and Future Works}

It is clearly shown in the study that the proposed BEE approach is a viable solution to the data sharing problem in an environment such as the e-Government where various concept domains are present simultaneously. It is also shown that the BEE approach complements the user requirement approach by correcting the flaws of the latter. Future works include additional researches in data structure and model, data architecture, and information system architecture based on the characteristics of individual layer.

\section{References}

[1] A. Gali, C. X. Chen and K. T. Claypool, "From Ontology to Relational Databases", ER Workshops LNCS, vol. 3289, (2004). 
[2] B. Otjacques, P. Hitzelberger and F. Feltz, "Interoperability of e-Government Information Systems: Issues of Identification and Data Sharing”, Journal of Management Information Systems, vol. 23, no. 4, (2007) Spring, pp. 29-51.

[3] C. H. Hwang, I. J. Son and H. Kyung Jung, "Study on Approach to Data Modeling for Sharing and Openness", Advanced Science and Technology Letters, vol. 44, (2013).

[4] H. Pundt and Y. Bishr, "Domain ontologies for data sharing-an example from environmental monitoring using field GIS", Computers \& Geosciences, vol. 28, (2002), pp. 95-102.

[5] K. Layne and J. W. Lee, "Developing fully e-Government: A four stage model", Government Information Quarterly, vol. 18, (2001), pp. 122 136.

[6] A. Kawtrakul, I. Mulasastra, T. Khampachua and S. Ruengittinun, "The Challenges of Accelerating Connected Government and Beyond: Thailand Perspectives", Electronic Journal of e-Government, vol. 9, issue 2, (2011), pp. 183-202.

[7] P. Pin and S. Chen, "The Entity-Relationship Data Model-A basis for the enterprise view of data", National Computer Conference, (1977), pp. 77-84.

[8] H. J. Scholl, "Interoperability in e-government: More than just smart middleware. In R.H. Sprague (ed.), Proceedings of the Thirty-Eighth Annual Hawaiian International Conference on System Sciences", Los Alamitos, CA: IEEE Computer Society Press, (2005).

[9] S. K. Milton and E. Kazmierczak, "An Ontology of Modeling Language: A Study Using a Common-Sense Realistic Ontology”, Journal of Database Management, (2007).

[10] T. Dillon, "Differentiating Conceptual Modeling from Data Modeling, Knowledge Modeling and Ontology Modeling and a Notation for Ontology Modeling", APCCM '08 Proceedings of the fifth Asia-Pacific conference on Conceptual Modelling, vol. 79, (2008), pp. 7-17.

\section{Authors}
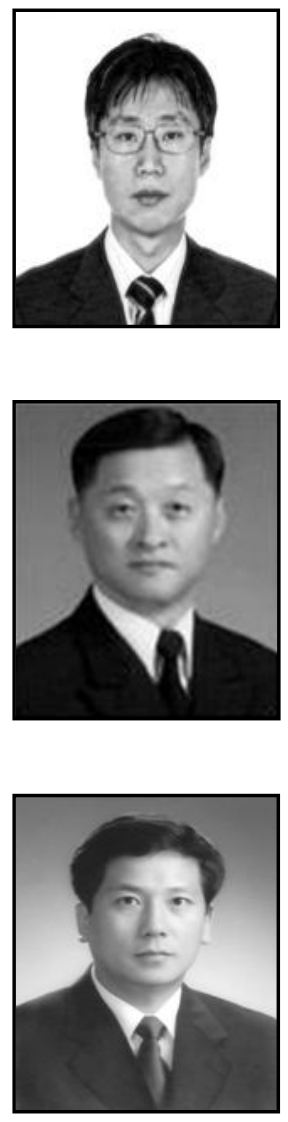

\section{Cheol Hyun Hwang}

He received the B.S degree from the Kumoh Institute Technology in 1991, and M.S. degree in Computer Science from Kyung-Nam University, and he is currently studying toward the Ph. D. degree in Computer Science at University of Paichai University. His current research interests include data modeling, master data, data architectures.

\section{Iek Jae Son}

He received the B.S degree from the Naval Academy of ROK and M.S. from Inha University in 1987. He is currently working chief of Naval Central Computing Center in South Korea. His current research interests include cyber-warfare, software engineering, database, security.

\section{Hoe-Kyung Jung}

He received the B.S degree in 1987 and $\mathrm{Ph}$. D. degree in 1993 from the Department of Computer Engineering of Kwangwoon University, Korea. From 1994 to 2005, he worked for ETRI as a researcher. Since 1994, he has worked in the department of Computer Engineering at Paichai University, where he now works as a professor. His current research interests include multimedia document architecture modeling, information processing, information retrieval, and databases. 